Research papers

\title{
Increased performance in the short-term water demand forecasting through the use of a parallel adaptive weighting strategy
}

\author{
A. Sardinha-Lourenço ${ }^{a}$, A. Andrade-Campos ${ }^{a, *}$, A. Antunes ${ }^{a}$, M.S. Oliveira ${ }^{b}$

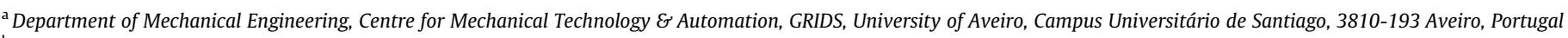 \\ ${ }^{\mathrm{b}}$ Department of Economics, Management, Industrial Engineering and Tourism, University of Aveiro, Campus Universitário de Santiago, $3810-193$ Aveiro, Portugal
}

\section{A R T I C L E I N F O}

\section{Article history:}

Received 29 November 2017

Received in revised form 19 January 2018

Accepted 21 January 2018

Available online 2 February 2018

This manuscript was handled by G. Syme,

Editor-in-Chief

\section{Keywords:}

Water demand

Forecasting

Adaptive weighting model

Short-term

\begin{abstract}
A B S T R A C T
Recent research on water demand short-term forecasting has shown that models using univariate time series based on historical data are useful and can be combined with other prediction methods to reduce errors. The behavior of water demands in drinking water distribution networks focuses on their repetitive nature and, under meteorological conditions and similar consumers, allows the development of a heuristic forecast model that, in turn, combined with other autoregressive models, can provide reliable forecasts. In this study, a parallel adaptive weighting strategy of water consumption forecast for the next 24-48 h, using univariate time series of potable water consumption, is proposed. Two Portuguese potable water distribution networks are used as case studies where the only input data are the consumption of water and the national calendar. For the development of the strategy, the Autoregressive Integrated Moving Average (ARIMA) method and a short-term forecast heuristic algorithm are used. Simulations with the model showed that, when using a parallel adaptive weighting strategy, the prediction error can be reduced by $15.96 \%$ and the average error by $9.20 \%$. This reduction is important in the control and management of water supply systems. The proposed methodology can be extended to other forecast methods, especially when it comes to the availability of multiple forecast models.
\end{abstract}

(C) 2018 Elsevier B.V. All rights reserved.

\section{Introduction}

The main objective in the management of drinking water distribution systems is to satisfy the demand of the consumers satisficing the continuous conditions of quality, flow, and adequate pressure thus ensuring a reliable distribution system. Efficiently managing and operating a potable water supply systems requires short-term forecasts of the water demand demanded by consumers (Adamowski et al., 2012; Coelho, 2016). The estimation of the future demand of water is fundamental for the planning of a regional system of water supply, since it will allow the reduction of costs (Zhou et al., 2002). These water demands are highly variable and fluctuate according to the type and size of the consumer, the time of day, the day of the week, the season of the year, the weather and even with the days of celebrations, extraordinary events and cost of supply of this service. Specific consumption patterns for each distribution network can be handled and interpreted skillfully by operators with large accumulated experience and used for operational control of the network. However, the use of these

\footnotetext{
* Corresponding author.

E-mail address: gilac@ua.pt (A. Andrade-Campos).
}

patterns is done in many cases manually, compromising efficiency and functioning of the network costs.

In the literature, it is possible to find numerous models developed by statistical methods, typically multiple regression and time series, to predict urban water consumption. For the daily operation of treatment plants and pumping stations, a short-term forecast model is needed for the next $24 \mathrm{~h}$ (Bakker et al., 2013a).

Many water demand forecasts models used in the literature use a one-hour step to study time series (Jowitt and Chengchao, 1992 Shvartser et al., 1993; Homwongs et al., 1994; Alvisi et al., 2007; Santos and Filho, 2014). These works show that it is possible to generate fairly accurate forecasts using as single input the historical demand (Msiza et al., 2008). Other forecast models include meteorological information as additional input. The model proposed by Zhou et al. (2002) uses the daily rainfall, the maximum temperature of the day, the number of days since the last rainfall and the effect of evaporation. The artificial neural networks (ANNs) model of Ghiassi et al. (2008) uses hourly temperature values and the ANNs model of Herrera et al. (2010) uses daily values of temperature, wind speed, atmospheric pressure, and rain.

Babel and Shinde (2011) evaluated the effect of weather variables as ANN inputs for daily and monthly water demand forecast in the city of Bangkok (Thailand). In the daily forecasts, 
no significant differences were found in the forecast accuracy when including weather variables (rainfall, average temperature, and relative humidity) in their models. In the work of Tabesh and Dini (2009), the best results were obtained with the ANN models and neural-fuzzy approaches considering only past water demand variables as input.

The quality and reliability of the input data for the analysis of a time series are crucial since it is the only information used to determine the future values. Critical Infrastructure Systems (CIS), including water distribution network systems, are large in size and occupy a large geographic space. These systems require a monitoring and control system in real time to ensure the maintenance of the variables in acceptable conditions for as long as necessary (Quevedo et al., 2010). Eventually, these variables suffer appreciable deviations due to failures (e.g. sensor and/or actuator and/or malfunctioning of pipelines) (Schütze et al., 2004). In the CIS, the telecontrol system has the function of acquiring, storing and validate data collected from different types of sensors in each time sample and thus monitoring in real time the whole system. Several problems can occur during the acquisition of process data, such as for example, those related to communication failure between sensors and the telecontrol system itself. These problems cause lost or damaged data. In that case, the missing data must be replaced by a set of estimated data, which should be representative of the data that was lost (Quevedo et al., 2010).

There are a large number of papers detailing various methodologies for short-term forecasts using different time scales: 15-min intervals (Bakker et al., 2013b); per hour (eg Shvartser et al., 1993; Zhou et al., 2002; Alvisi et al., 2007) as well as daily/monthly time scales (Maidment and Parzen, 1984a; Franklin and Maidment, 1986; Smith, 1998; Miaou, 1990). In all these documents, recurrent patterns and the periodicities that exist in the data of water demand, at different levels of temporal aggregation, were recognized.

Most models of prediction of water demand have a limited number of demand patterns. Jowitt and Chengchao (1992) and Homwongs et al. (1994) used three different water consumption patterns for the development of their model: one for weekdays, one for Saturdays and one for Sundays. The model described by Zhou et al. (2002) uses only two different patterns: working days and another for national holidays and weekends. Alvisi et al. (2007) propose the identification of the patterns implicit in the time series of the water demand of the Castelfranco Emilia municipality, Italy. The study was conducted for daily water demand for a whole year, finding that the demands increase during the summer period and during the week from Sunday to Saturday. In the same way, the demands of water per hour show a variable behavior during the day, with different patterns depending on if it is a weekday or weekend. According to their analysis, the daily and hourly series observed present a pattern of demand during the holidays very similar to the one observed during the weekends.

Recently, Bakker et al. (2013b) proposed a model that uses a relatively greater number of demand patterns, discern not only the demand patterns for the seven days of the week, but also for a series of day types that deviate from the common days of the week, such as primary school vacation periods and private day events related to multiple activities for the Netherlands. In this study, national holidays are treated as Sundays.

The developed heuristic model predicts water demand for the next $48 \mathrm{~h}$ with 15 -min steps. The model determines water demand in three main phases: in the first phase, the average water demand is forecasted for the next $48 \mathrm{~h}$. In the second phase, normal water demands are anticipated for individual steps of $15 \mathrm{~min}$. In the third phase, if necessary, additional water demands are anticipated for reasons of increasing the ambient temperature (summer) for the individual 15-min stages.
The work of Bakker et al. (2013b) focuses on the repetitive nature of the behavior of water demands in distribution networks. For the development of an adaptive heuristic forecast model, he used a large amount of historical data from the water distribution networks for conditions and type of similar consumers, allowing him to determine particular parameters of daily behavior for his forecast model. For the calculation of these parameters, the model requires many days of historical information, which represents a disadvantage if consumption undergoes shorter periodic variations in its behavior and there is a large amount of continuous historical information validated.

Winkler and Makridakis (1983) affirm that the combination of forecasts improves accuracy and using simple averages, in the combination of forecasts, also reduces the variability of the errors in the forecast and, therefore, the risk associated with the choice of forecasting method. Recent research on short-term demand forecasting has shown that models using univariate time series based on historical data are useful and can be combined with other prediction methods to reduce errors. Therefore, the forecasts can be combined by using simple and optimal weights.

Stock and Watson (1999) proposed several pooling procedures that differ by the amount of weight placed on the model as a function of the currently best performance. These procedures include the equally weighting of all the forecast, the weighting inversely proportional to their current mean squared error (MSE), using average forecast, and placing all weight on the forecasting method that currently has the lowest simulated real-time MSE. The final pooling procedure is simulated using real-time model selection.

The methodology used in Caiado (2010) was to weight the forecasts of three models with the inverse of the mean squares errors (MSE) of each of the individual methods. The average error difference when using individual prediction methods to predict a single day was $8.33 \%$ greater than combining forecasting methods. In the prediction of 2 and 3 days, combined forecasts can reduce error by $12.77 \%$ and $10.64 \%$, respectively (Caiado, 2010).

In this study, a parallel adaptive weighting model of water consumption forecast for the next $24-48 \mathrm{~h}$, using univariate time series of drinking water consumption, is proposed. For the development of the model, it is used a seasonally integrated autoregressive integrated mobile averages (ARIMA) and a shortterm forecasting heuristic method (Bakker et al., 2013a,b), which takes into account the multiple daily water consumption patterns. This last method classifies the historical information of the water demands of the working days and the typical days and then makes the forecasts. A third method is developed from the classification made previously and the use of the ARIMA methodology. Adaptive weighting parameters are calculated for each one of the 24-48-h parallel forecasts methods according to the minimum mean absolute percentage error (MAPE) obtained for the day previous. The model was validated using Portuguese networks as case studies.

This forecasting strategy that uses a combination of optimal weights allows the proposed model to be adaptive, simple to implement and scalable for the incorporation of a larger number of forecast algorithms.

\section{Water demand forecasting}

\subsection{Time series analysis}

The temporal series are generally analyzed from a deterministic point of view. However, nowadays, time series are also studied from a stochastic point of view, using more complex methods and their application requires larger data. Statistical models for time series were developed by Box and Jenkins (1976) that consider the dependence between the data. The analyses are based 
on an explicit model. The models are known by the generic name of ARIMA (Autoregressive Integrated Moving Average), describing a value as a linear function of previous data and random errors and may include a cyclical or seasonal component.

Non-seasonal ARIMA models are usually called ARIMA $(p, d, q)$ where $p, d$ and $q$ are non-negative integers. $p$ is the order (number of delays) of the autoregressive model, $d$ is the degree of differencing (the number of times the data have had past values subtracted), and $q$ is the order of the moving average model. The ARIMA seasonal models are written as ARIMA $(P, D, Q) m$, where $m$ refers to the number of seasonal periods and the capital letters $P, D, Q$ refers, likewise, autoregression, differentiation, and terms of the moving average for the seasonal part. A simple approach to a seasonal ARIMA model is to model the regular and seasonal dependency separately, and then build the model incorporating these parts in a multiplicative way. Thus, a seasonal ARIMA model has the form:

$\Phi_{P}\left(B^{S}\right) \phi_{p}(B) \nabla_{s}^{D} \nabla^{d} z_{t}=\theta_{q}(B) \Theta_{Q}\left(B^{S}\right) \alpha_{t}$,

where $z_{t}$ is the original value of the time series, $\Phi_{P}\left(B^{S}\right)=\left(1-\Phi_{1} B^{S}-\ldots-\Phi_{P} B^{S P}\right)$ is the seasonal autoregression operator of order $P, \phi_{p}=\left(1-\phi_{1} B-\ldots-\phi_{P} B^{p}\right)$ is the regular autoregression operator of order $p, \nabla_{s}^{D}=\left(1-B^{s}\right)^{D}$ represent the seasonal differences, $\nabla^{d}=(1-B)^{d}$ is the regular differences; $\theta_{q}(B)=\left(1-\theta_{1} B-\ldots-\theta_{q} B^{q}\right)$ represent the regular moving average operator of order q, $\Theta_{Q}\left(B^{S}\right)=\left(1-\Theta_{1} B^{S}-\ldots-\Theta_{Q} B^{s Q}\right)$ is the seasonal moving average operator of order $Q$ and $\alpha_{t}$ is a random error or white noise process.

\subsection{Heuristic forecasting model}

The heuristic model determines the demand patterns and factors related to the day type and demand of the day using the water consumption according to a pre-established number of historical data (Bakker et al., 2013a,b). While it is being executed, the model constantly renews these patterns and factors. In this way, the model automatically adapts to the characteristics of water demand to generate forecasts every $48 \mathrm{~h}$. This functionality allows using the model throughout the year, and not using different configurations for each season. The model not only distinguishes demand patterns for the seven days of the week, but also for national holidays. These national holidays are treated as Sundays (Bakker et al., 2013a,b).

The model forecasts the water demand for the subsequent $48 \mathrm{~h}$ with a time step of one hour. However, the calculation is done every day at 00:00 h, updating the $0-24 \mathrm{~h}$ forecasts and calculating the new $24-48 \mathrm{~h}$ forecasts. These forecasts are stored together with the historical data.

The heuristic model predicts the demand for water in two stages: in the first stage, the average flow of water is forecasted for the next $48 \mathrm{~h}$; in the second stage, the typical water flows are determined for each hour according to the day to forecast (Bakker et al., 2013a,b).

The forecast of the average flow of water for the subsequent 48 $\mathrm{h}$ is done using the water demand measured in the previous $48 \mathrm{~h}$. To correct the influence of the day of the week, the water demand measured for each hour $\left(W D_{t}\right)$ is divided by a typical factor that corresponds to the day of the week $\left(f_{\mathrm{dw}, i}\right)$. The corrected water demand $\left(W D_{\text {corr }, t}\right)$ is calculated through

$W D_{\text {corr }, t}=\frac{W D_{t}}{f_{\mathrm{dw}, i}}$

The typical factors of the day of the week $\left(f_{\mathrm{dw}, i}\right)$ for each type of day $t_{i}$ are calculated in a time window of $m$ previous observations of water demand, given as $f_{\mathrm{dw}, i}=\frac{\frac{1}{m} \sum_{i=1}^{i=m} W D_{\mathrm{avg}, \mathrm{dw}, i}}{\frac{1}{7 m} \sum_{i=1}^{i=7 m} W D_{\mathrm{avg}, \mathrm{all}, i}}$,

where $W D_{\text {avg,dw,i }}$ is water demand average for equal day type and $W D_{\text {avg.all,i }}$ is the water demand day for the window observation. By default, $m=10$ (Bakker et al., 2013a,b). For example, to determine the Sunday typical day factor, the average consumption of the previous ten Sundays is calculated and divided by the average of all the daily consumptions of the last seventy days.

In order to determine the average predicted water demand $\left(W D_{\text {forc.avg }}\right)$ during the next $48 \mathrm{~h}$, the water demand for the previous $48 \mathrm{~h}$ is weighted according to equation following (first day: $t=-23,0$; second day: $t=-24,-48 \mathrm{~h}$ ):

$$
W D_{\text {forc avg }}=C 1\left(\frac{1}{24} \sum_{t=-23}^{t=0} W D_{\text {corr }, t}\right)+C 2\left(\frac{1}{24} \sum_{t=-48}^{t=-24} W D_{\text {corr }, t}\right)
$$

The constants $\mathrm{C} 1$ and $\mathrm{C} 2$ are set by default at 0.85 and 0.15 respectively (Bakker et al., 2013a,b). According to the criterion of Bakker et al. (2013a,b), the most recent water measurements have greater weight than the older demands. Given a change in the consumption pattern or the value of demand, a rapid adjustment of forecast water demand would be obtained.

The hourly demand factor is calculated through the averages of the hourly water demands of the last 5 typical days (Bakker et al., $2013 a, b)$. The hourly factor $\left(f_{\text {hour } i}\right)$ is determined by the ratio of water consumption for each hour between the average consumption obtained during the day:

$f_{\text {hour }, i}=\frac{W D_{t, i}}{\frac{1}{24} \sum_{i=-23}^{i=0} W D_{t, i}}$.

In step two, the future water demands $\left(W D_{\text {forc }, t}\right)$ are calculated for the next $48 \mathrm{~h}$. This is achieved by multiplying forecasts average demand $\left(W D_{\text {forc,avg }}\right)$ for the typical factor of the day of the week $\left(f_{\mathrm{dw}, i}\right)$ and the typical factor hourly for to forecast day $\left(f_{\mathrm{hour}, i}\right)$ :

$W D_{\text {forc }, t}=W D_{\text {forc }, \text { avg }} f_{\mathrm{dw}, i} f_{\text {hour }, i}$.

\section{Drinking water demand forecasts: Parallel adaptive weighting strategy (PAWS)}

The ARIMA and ANN models have gained wide acceptance as successfully predictive models in linear or nonlinear domains. However, no model is universally suitable for all time series. The idea of combining models to generate forecasts is to use the best features of each model to capture the different patterns in the data. Both theoretical and empirical data suggest that the combination of methods can be an effective and efficient way to improve predictions (Zhang, 2003). The heuristic models have been developed for specific applications, since they present peculiarities of the studied system, such as the empirical model developed for drinking water consumption forecasts by Bakker et al. (2013b).

In this work, we propose a Parallel Adaptive Weighting Strategy (PAWS) to the forecasting of drinking water demand. To validate this strategy, both the ARIMA model and a heuristic model based on historical consumption are used. The motivation of the PAWS model is based on the fact that it is often difficult to determine in practice if a time series presents a linear or nonlinear behavior and, in general, if a particular method is more efficient than another. It is very difficult for forecasters to choose the correct technique for their cases studies. In a real-time case, since it is difficult to analyse the characteristics of the data, forecasting using different models (ARIMA, heuristics, etc.) and a weighting strategy can be a good practical approach. Zhang (2003) developed a hybrid model combining ARIMA and ANN models, using the ARIMA model 
to analyze the linear part of the time series and the neural network model to capture the residues of the ARIMA model. It may be reasonable then to consider a time series composed of a linear autocorrelation and a structure with a nonlinear component.

The proposed strategy, PAWS, combines different models used in the determination of water demand forecasts, assigning them a weight according to their degree of particular deviation with respect to the actual value of demand. For this study, we worked with three methods: a heuristic and two ARIMA methods (with and without classification of the typical days). That is,

$\widehat{W D}_{\mathrm{PAWS}, t}=\sum_{i=1}^{n} W D_{\mathrm{M}_{i}, t} k_{i}$ with $\sum_{i=1}^{n} k_{i}=1$ and $t=1, \ldots, n_{\text {steps }}$

where $\widehat{W D}_{\text {PAWs,t }}$ denotes the predicted value of water demand for time $t, W D_{\mathrm{M}_{i}, t}$ the water demand found by each model, $n$ is the number of forecasting models used in the parallel strategy and $n_{\text {steps }}$ are the number of time steps used. The parameters $k_{i}$ are the weighting coefficients whose values vary between 0 and 1 . These parameters must be found in such a way as to minimize the error between the observed data series and the predicted values. Independently of the metrics of the errors used $\left(R^{2}\right.$, MAPE, MAE, etc.), errors and predicted value are calculated for each model forecast using the historical data of the demands during a given period. In this work, the weighting parameters are obtained according to the historical success of each model used, particularly according to recent historical success. This paper proposes, as a first strategy, a methodology for the identification of atypical values and the validation of raw data of water consumption. Then, the classification of typical days of the week and holidays as a preliminary step for the calculations of two forecasting algorithms is made: a heuristic model and another autoregressive model. Finally, the determination of the weighting parameters for the weighted calculation of the forecast and then be used to generate the definitive $24-48 \mathrm{~h}$ forecast of the water demand. The weights $k$ that minimize the MAPE error function are determined solving an optimization problem using the calculated errors for every hour of the last day before the forecast. That is,

Find $k_{i}, i=1, \ldots, n$, in order to

minimize $f\left(k_{i}\right)=\frac{1}{24} \sum_{t=1}^{t=24} M A P E_{t}=\sum_{t=1}^{t=24} \frac{100}{24} \sum_{i=1}^{n} \frac{y_{t}-W D_{M_{i}, t} k_{i}}{y_{t}}$,

subject to $0 \leqslant k_{i} \leqslant 1$, and $\sum_{i=1}^{i=n} k_{i}=1$.

In Fig. 1, a scheme of the Parallel Adaptive Weighting Strategy model is presented.

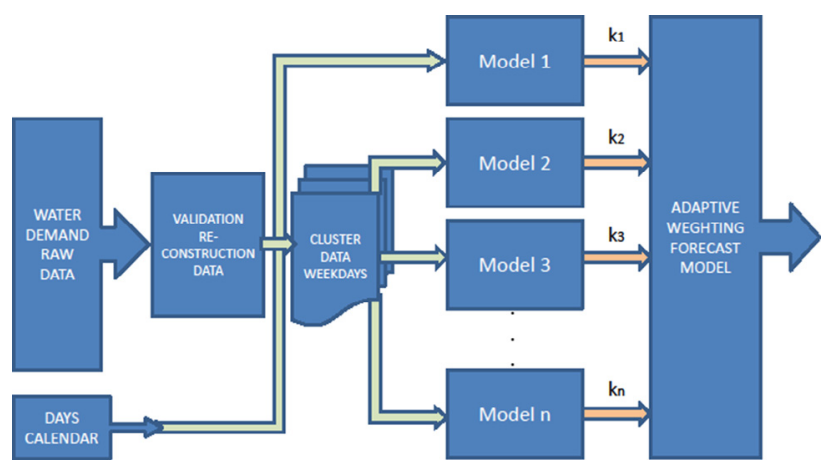

Fig. 1. Scheme of the Parallel Adaptive Weighting model for water demand forecast.

\subsection{PAWS model using ARIMA and a heuristic model}

For the development of the ARIMA model, the methodology proposed by Box-Jenkins is used. This approach provides the tools to identify suitable time series models by comparing the behavior of the time series data with the autocorrelation function (ACF) and Partial autocorrelation function (PACF). This methodology is applied to stationary time series, that is, its mean and variance are constant or fluctuate little in the time interval analyzed. Otherwise, it is necessary to make the pertinent modifications and adjustments (differentiation) of the data to achieve stationarity. If the original values of the time series are non-stationary and seasonal, the most complex differentiation transformations are necessary. Once the ARIMA model is identified, the characteristic parameters $(p, d, q)$ or $(p, d, q)(P, D, Q)_{m}$ are determined for the seasonal or non-seasonal model, respectively. The Akaike information criterion (AIC) is used to check and select the forecasting model. The aim of this criterion is to find the simplest model possible and prevent over-fitting (Bennett et al., 2013). To evaluate the predictive performance of the different models, each data set is divided into two samples: one for training and other for evaluation or testing. The training data set is used to generate the model and then the remaining sample of data is used to evaluate the established model. The set of historical data was classified according to the types of days of the week and national holidays, generating two batches of potential data: a batch of data divided by clusters and another batch of unclassified data. Each of them was used as input for two autoregressive ARIMA models and, therefore, to generate a forecast for each batch of data.

The development of the forecast heuristic model is based on the model proposed by Bakker et al. (2013b). The heuristic model proposed in this work is developed from the information provided by two Portuguese potable water distribution networks that simultaneously serve the residential, industrial and agricultural sectors.

The case studies used in this document correspond to multimunicipal water supply subsystems of the central-north region of the country. The systems are responsible for supplying flows ranging from 0.2 to $240 \frac{\mathrm{Mm}^{3}}{\mathrm{day}}$. The information provided consists of water consumption data measured at time intervals of one hour. Therefore, the constructed model uses this sampling time.

The effect of combining large numbers of consumers generates a behavior profile that is reflected in well-defined patterns (Zhou, 2002). Figs. 2 and 3 shows a pattern of daily drinking water consumption for a subsector of water supply systems of central of Portugal, serving a population of 15,972 inhabitants.

In general, water consumption patterns show a mild random component for a similar daily pattern during the days of the week and one for Sundays and Saturdays. It is also possible to appreciate the change in pattern and consumption values for the hot and cold months.

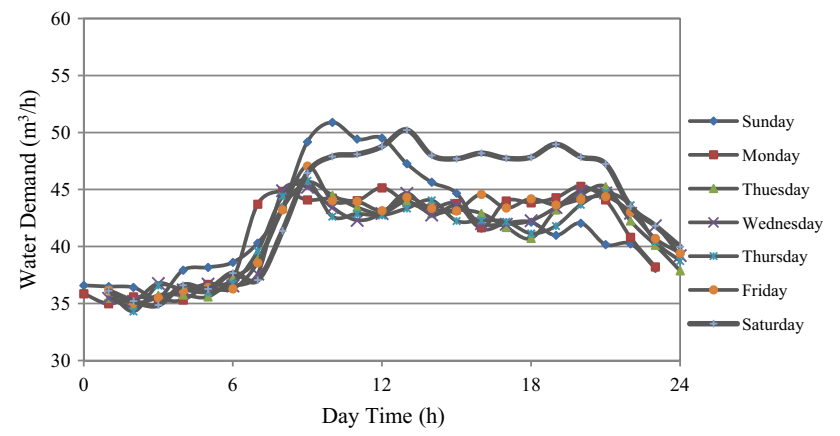

Fig. 2. Average daily profile water consumption for a water supply system of central of Portugal area (based on available hourly data of the cold months of 2016). 


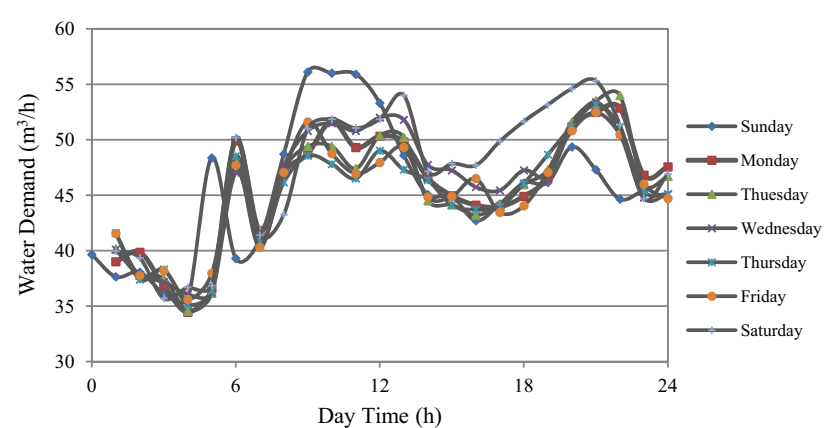

Fig. 3. Average daily profile water consumption for a water supply system of central of Portugal (based on available hourly data of the warm months of 2016).

\subsection{Setup of the model}

For the implementation of the model, the measured water demands and the calendar data were used as the only inputs of data to organize and classify different types of the day of the week, as well as national holidays. The model does not require any weather information. Although the relationship between water demand and the atmospheric conditions seems obvious, the model does not use the climatic variables. However, gradual climate changes (summer-winter) are observed and are manifested in the pattern of seasonal consumption where it is captured through the historical data record and are then used to generate consumption forecasts.

The validation of the proposed model initially determines three forecasts according to the calculation scheme adopted: two forecasts are calculated using the ARIMA model, one with the unclassified data set and another with the classified ones. The third forecast is calculated according to a heuristic model that determines the demand pattern and factors related to the day type and demand of the day, using the water consumption according to a pre-established number of historical data. While running, at every $24 \mathrm{~h}$, the heuristic model constantly renews these patterns and factors with the incorporation of new historical demand data. In this way, the model automatically adapts to the characteristics of water demand to generate $48 \mathrm{~h}$ forecasts every $24 \mathrm{~h}$. This functionality allows using the model throughout the year, and not using different configurations for each season. The model not only distinguishes demand patterns for the seven days of the week, but also for national holidays. These national holidays are treated as Sundays.

This paper also proposes a methodology based on the reconstruction and validation of raw data based on statistical concepts (Coefficient of Variance, Standard Deviation, and Normal Distribution) and the methodology of validation proposed by Quevedo, et al. (2010).

All the forecasting models described in the PAWS model formulation were implemented in the Python programming language in its version 3.6.

\subsection{Forecasting error metrics}

The determination of errors in forecasting is crucial for the selection of the model parameters as well as for monitoring the accuracy and reliability of the forecasts generated. Many researchers have proposed several measures, but the most widely adopted in the forecasting of water demand is the mean absolute percentage error (MAPE) (Hyndman and Athanasopoulos, 2013; Bakker et al., 2013b; Sampathirao et al., 2014; Bai et al., 2015; Coelho, 2016; Candelieri, 2017), expressed as:
$M A P E=\frac{100}{n} \sum_{t=1}^{t=n}\left|\frac{y_{t}-W D_{\mathrm{M}, t}}{y_{t}}\right|$,

where $y_{t}$ is the demand of water observed at time $t, W D_{\mathrm{M}, t}$ is the forecast water demand of model at time $t$ and $n$ is the length of the evaluated times series. In many research studies where time series forecasts are calculated, the $\mathrm{R}^{2}$ coefficient and the RMSE is also usually evaluated (Bennett et al., 2013).

\section{Benchmark}

It is a good practice for each new forecasting method or strategy to be compared with a confirmed study using known benchmarks to check its strengths, potentialities and possible limitations. For univariate time series methods, it is advisable to make comparisons at least with a standard method such as the ARIMA model. The first benchmark, described in Appendix A, is a time series generated by sine functions in order to obtain a cyclical behavior to represent the demand for drinking water daily, weekly and annual patterns. Noise was added to the time series using a random variable. The dataset generated, shown in Fig. 4, contains 17,544 values in one-hour intervals.

The second benchmark is an air quality dataset. The dataset contains 9358 instances of hourly averaged responses from an array of 5 metal oxide chemical sensors embedded in an Air Quality Chemical Multisensor Device (Vito et al., 2008). For this study, only one variable was selected: Total Nitrogen Oxides (NOx). Fig. 5 shows the concentration of NOx for one week.

The benchmarks were used to evaluate the proposed PAWS strategy and were compared with the simple Mean weights strategy and the Mean Square Error (MSE) Weights strategy proposed by Stock and Watson (1999). In the mean weighting scheme, equal contribution was considered for each forecast model. The MSE weights strategy corresponds to Bates and Grangers (1969) suggestion, where the weights are inverse to the mean square error (MSE).

For the benchmarks datasets, each data set is divided into two samples: (1) training and (2) evaluation or testing. The training data set is used to generate the model and the remaining data is used to evaluate the established model by the calculation of the forecasting error. The proposed PAWS strategy used two models based on the ARIMA model and a heuristic model to calculate forecasts.

The Box-Jenkins methodology for the ARIMA model requires at least 50 values. Multiple groups of $144 \mathrm{~h}$ (one week) are used as training data set and the MAPE error is measured every hour in a forecast of $48 \mathrm{~h}$. The computational time used by the developed algorithm was also measured.

According to a previous analysis made for the selected benchmark sets, the training sample should be $336 \mathrm{~h}$. For larger samples, the error is substantially increased due to overtraining. Fig. 6 shows the results obtained with the ARIMA model for benchmark 1 .

The forecast values were evaluated for the last two days of the data set. The errors obtained for each benchmark are listed in Table 1.

Table 1 shows a poor performance of the forecast models for benchmark 2 due to the absence of temporary patterns and the presence of outliers. However, the PAWS strategy yields better results.

\section{Case study results}

For this work, two water supply companies from central and northern Portugal are used as case studies. The data provided 


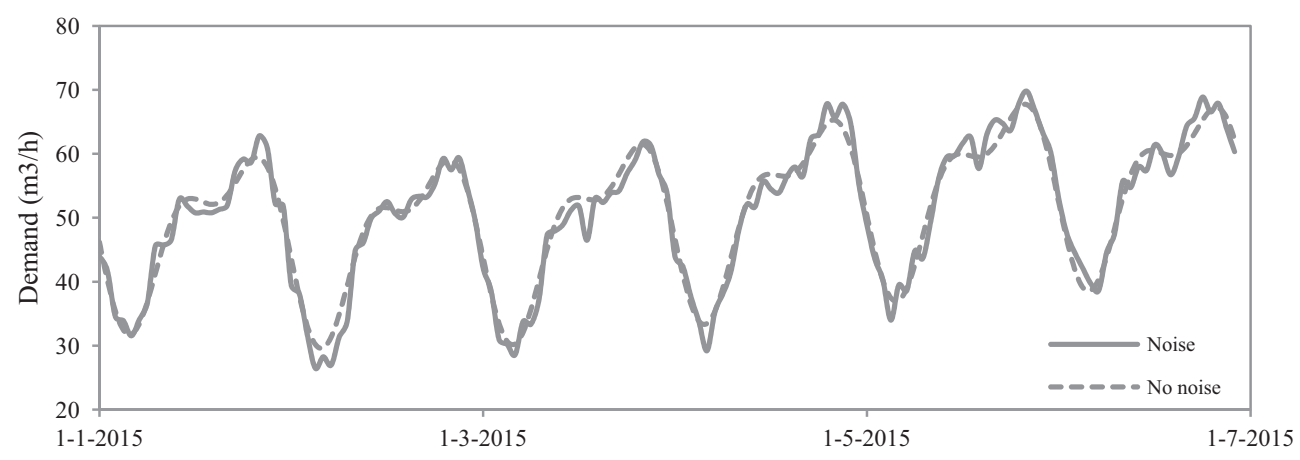

Fig. 4. Benchmark 1: Seasonal time series generated for one week (144 h).

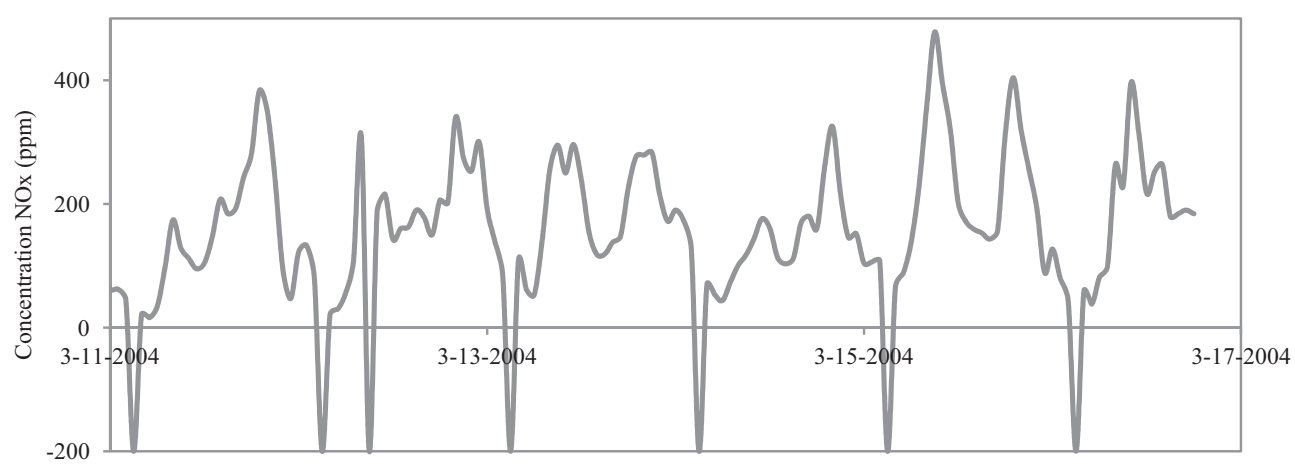

Fig. 5. Benchmark 2: Concentration of NOx for one week (144 h). Missing values are tagged with the -200 value.

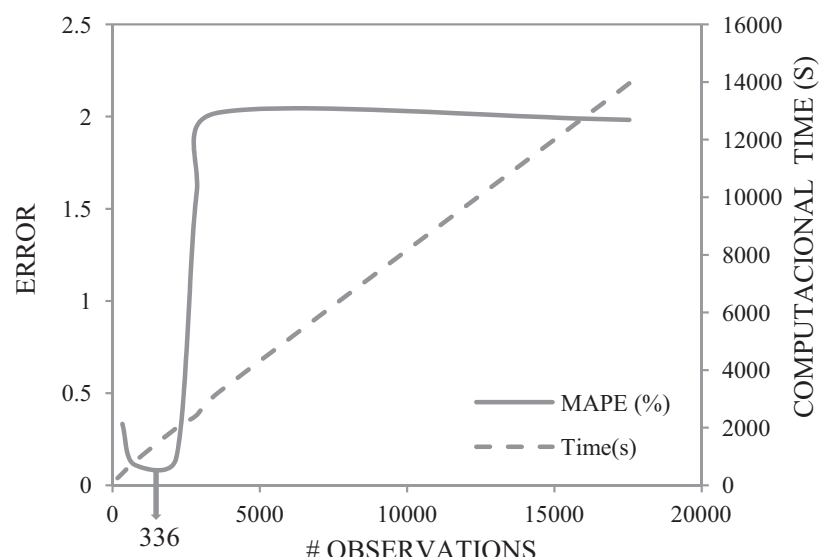

Fig. 6. Forecasting errors and computational time obtained with the number of observations used to develop the ARIMA model for benchmark 1.

Table 1

Errors calculated for benchmarks.

\begin{tabular}{llll}
\hline Benchmark & Forecasting Model & $\mathrm{R}^{2}$ & MAPE (\%) \\
\hline 1 & ARIMA & 0.9997 & 1.961 \\
& ARIMA clustering & 0.9986 & 1.853 \\
& Heuristic & 0.9830 & 1.597 \\
& Mean weights & 0.999 & 2.241 \\
MSE Weights & 0.999 & 1.318 \\
& PAWS & 0.9997 & 0.856 \\
2 & ARIMA & 0.4779 & 76.855 \\
& ARIMA clustering & 0.3556 & 88.658 \\
& Heuristic & -0.0765 & 68.133 \\
& Mean weights & 0.571 & 191.300 \\
& MSE Weights & -0.209 & 50.861 \\
& PAWS & 0.4879 & 35.402 \\
\hline
\end{tabular}

includes water demands for continuous periods of 1-h intervals for a period of approximately 1 year. The data corresponds to sub-areas of the system where there are different types of consumers: domestic, industrial and agricultural. In the case of the northern network, WD2, WD4 and WD16 report for the period of August 2012 to July 2013 (Coelho, 2016). For the central network of Portugal, WDa, WDb and WDc report to the period of 2015 and 2016.

\subsection{Input data analysis}

The received raw data presented multiple acquisition errors. With the analysis of the chronological charts of the raw data provided by water service companies (see Fig. 7), it was clear that, in most cases, several failures occurred. The data was provided as cumulative total volumes as a function of time, which were then transformed into instantaneous flows or water demands.

Another typical problem in raw data is related to the absence of information in certain periods. To solve this problem, the missing values are estimated with historical data. For this work, a maximum time of 48 continuous hours is proposed. Fig. 8 shows a set of water consumption data that were refined and reconstructed using the proposed methodology.

The analysis method used in the Northern Portugal network was based on the interquartile range of each data set. However, all the data used was subjected to the mentioned analysis of validation and reconstruction. Although the identification of the missing and atypical data represented a small amount (smaller than $3 \%$ ), the statistical analysis of the data reflects a significant variation particularly in the standard deviation of WDc, due to the maximum and minimum values replacement. In the case of time series, specifically for the demands of drinking water, all values should be positive, due to the constant water demand of the consumers and the presence of leaks in the system. Table 2 shows the water 

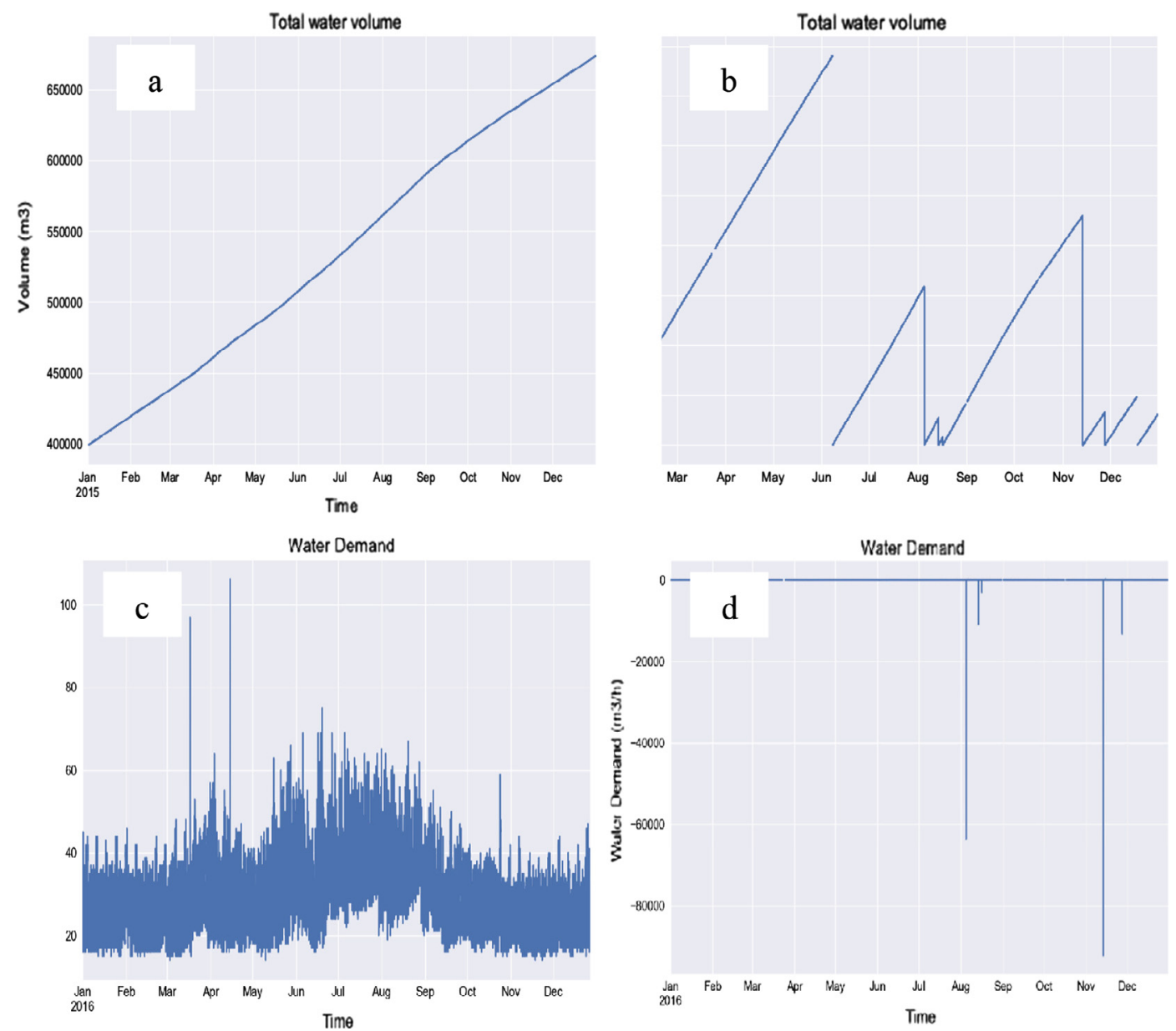

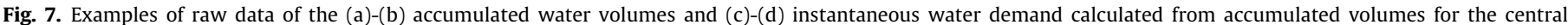
Portugal water network.

demands studied and the statistical information for each of the case studies before and after of validation, respectively.

\subsection{Clustering weekdays data}

For the developed forecast calculation model it is necessary to classify the consumption according to the calendar day and to its pattern of hourly behavior and, consequently, to decide if it can be considered as a predictor variable included in the model. After analyzing the patterns revealed by the time series plots, it was verified that for different months, different patterns were presented, as well as different days of the week and national holidays.

An analysis of the influence of the anthropic variables (month, day and time of day) through the Pearson correlation index is a method that helps to make a classification of the chronological data set. Tables 3 show the results of the Pearson index for water demands of each of the water supply systems studied (WDa with shading and WD4 without shading).

It can be seen that the days of the week present a high correlation between them, which allows classifying these days as common. Days Saturdays and Sundays present a high correlation between them, but for classification purposes, it was decided to keep them separated due to the uneven pattern in the morning. The holidays show a correlation index lower than the others. This indicates that it belongs to a different group of days. However, due to the separation between the different dates of these days that affect the consumption patterns of the warm and cold months, it was decided to include it in the classification of the Saturdays due to its high correlations. The results for the other time series studied show similar results.

The behavior is similar in both the cold and warm months although the daily patterns do present variations especially in the early hours of the morning (Figs. 2 and 3). In the summer months, the water consumption increases with respect to the winter period. This behavior manifests itself in a gradual and slow way, which can be captured through the historical information of the demands and then used to generate short forecasts term. Fig. 9 shows the daily consumption patterns for the month of December and August 2015 for the WDa water demand and the daily consumption pattern for the weekends and the Christmas day holiday. This behaviour is very similar to the other time series studied.

\subsection{Water demand forecasting}

The validation of the PAWS model uses three short-term forecasts: two forecasts are calculated using the ARIMA model. One of the unclassified data set and the other with the classification according to the type of day. The third forecast is calculated according to a heuristic model. The calculation of the final forecasting is done with the determination of the weights of the $k$ parameters that minimize the MAPE.

According to the Box-Jenkins methodology, it was verified that the time series were stationary. The characteristic parameters 


\section{Water Demand "Debug data validated"}
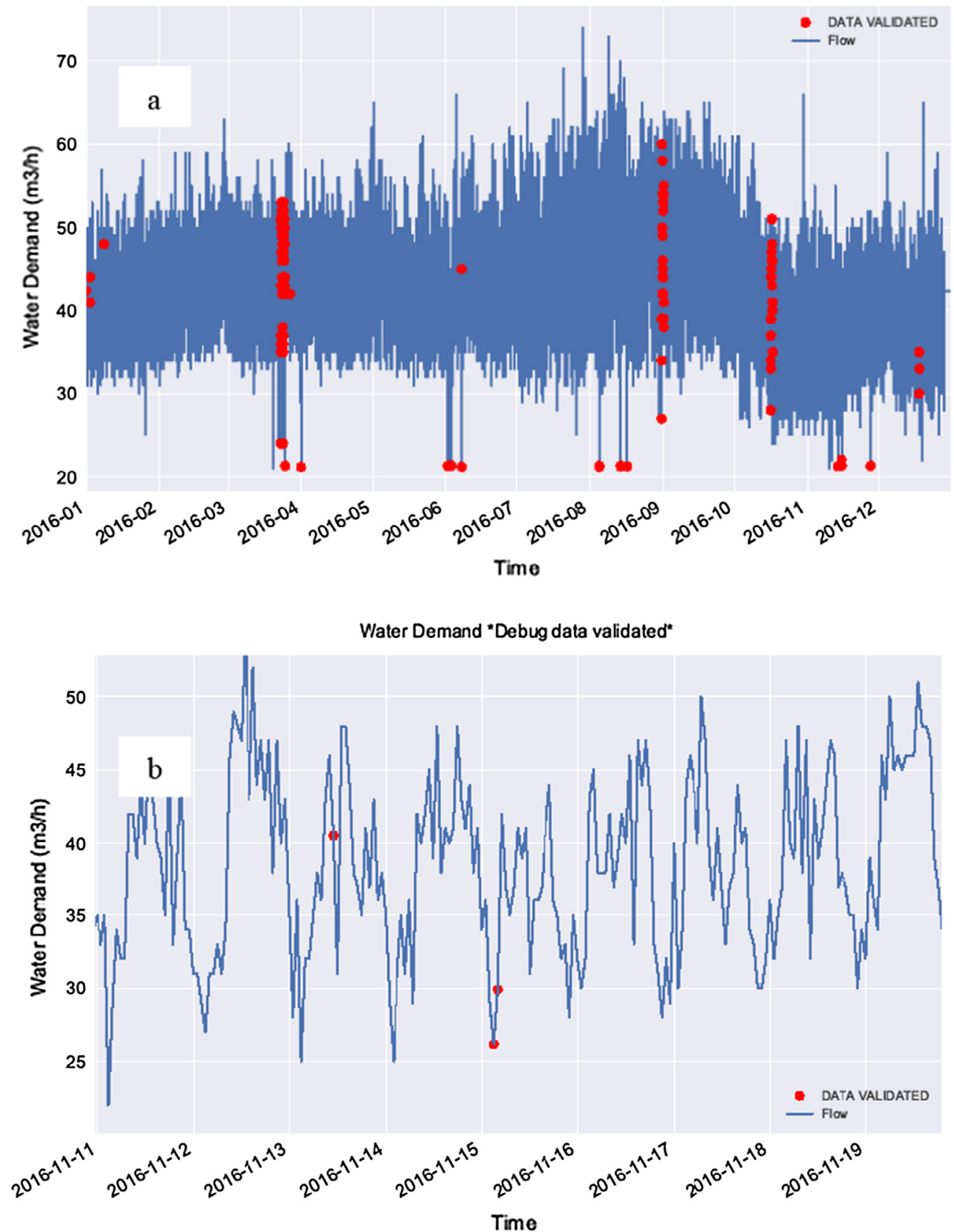

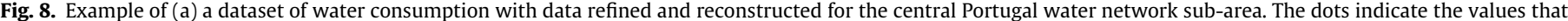
were validated. (b) A dataset of water consumption with data estimated for a period of 11-19 November 2016.

Table 2

Statistical information on water demands before and after validation of data for the two regions of the country.

\begin{tabular}{|c|c|c|c|c|c|c|c|}
\hline \multirow{2}{*}{\multicolumn{2}{|c|}{$\begin{array}{l}\text { Drinking water system of Portugal } \\
\text { Water demand }\end{array}$}} & \multicolumn{3}{|c|}{ Northern } & \multicolumn{3}{|l|}{ Central } \\
\hline & & $\begin{array}{l}\text { WD2 } \\
7536\end{array}$ & $\begin{array}{l}\text { WD4 } \\
7536\end{array}$ & $\frac{\text { WD16 }}{7536}$ & $\frac{\text { WDa }}{8760}$ & $\frac{\mathrm{WDb}}{7320}$ & $\begin{array}{l}\text { WDc } \\
8760\end{array}$ \\
\hline Before validation & $\begin{array}{l}\text { Total Observations } \\
\text { Mean }\left(\mathrm{m}^{3} / \mathrm{h}\right) \\
\text { Standard Deviation }\left(\mathrm{m}^{3} / \mathrm{h}\right) \\
\text { Minimum }\left(\mathrm{m}^{3} / \mathrm{h}\right) \\
\text { Maximum }\left(\mathrm{m}^{3} / \mathrm{h}\right)\end{array}$ & $\begin{array}{l}7536 \\
11.62 \\
5.30 \\
0.00 \\
34.67\end{array}$ & $\begin{array}{l}7536 \\
37.63 \\
16.80 \\
0.00 \\
97.71\end{array}$ & $\begin{array}{l}7536 \\
2.67 \\
1.82 \\
0.00 \\
14.42\end{array}$ & $\begin{array}{l}8760 \\
31.37 \\
9.01 \\
14.00 \\
106.00\end{array}$ & $\begin{array}{l}7320 \\
8.84 \\
2.97 \\
4.20 \\
27.60\end{array}$ & $\begin{array}{l}8760 \\
22.02 \\
1219.64 \\
-92201.00 \\
240\end{array}$ \\
\hline After validation & $\begin{array}{l}\text { Mean }\left(\mathrm{m}^{3} / \mathrm{h}\right) \\
\text { Standard Deviation }\left(\mathrm{m}^{3} / \mathrm{h}\right) \\
\text { Minimum }\left(\mathrm{m}^{3} / \mathrm{h}\right) \\
\text { Maximum }\left(\mathrm{m}^{3} / \mathrm{h}\right) \\
\text { Outliers } \\
\text { \% Outliers }\end{array}$ & $\begin{array}{l}11.62 \\
5.26 \\
0.12 \\
28.96 \\
23 \\
0.31\end{array}$ & $\begin{array}{l}37.63 \\
16.80 \\
1.29 \\
92.92 \\
10 \\
0.13\end{array}$ & $\begin{array}{l}2.67 \\
1.80 \\
0.04 \\
8.46 \\
21 \\
0.28\end{array}$ & $\begin{array}{l}31.33 \\
8.85 \\
14.00 \\
58.40 \\
71 \\
0.81\end{array}$ & $\begin{array}{l}8.69 \\
2.38 \\
4.20 \\
16.06 \\
218 \\
2.49\end{array}$ & $\begin{array}{l}43.18 \\
7.28 \\
21 \\
74 \\
238 \\
2.72\end{array}$ \\
\hline
\end{tabular}


Table 3

Pearson correlation index for drinking water system of central Portugal (up-right side of the matrix, grey area) and northern Portugal (down-left side).

\begin{tabular}{lcccccccc}
\hline \multicolumn{7}{c}{ Weekdays Pearson correlation index (WDa and WD4) } \\
& Sunday & Monday & Tuesday & Wednesday & Thursday & Friday & Saturday & Holidays \\
\hline Sunday & -- & 0.7482 & 0.7231 & 0.7182 & 0.7075 & 0.6999 & 0.8230 & 0.5418 \\
Monday & 0.7865 & -- & 0.8948 & 0.8887 & 0.7912 & 0.8437 & 0.7944 & 0.5030 \\
Tuesday & 0.7630 & 0.9456 & -- & 0.9068 & 0.7937 & 0.8745 & 0.7955 & 0.5030 \\
Wednesday & 0.7664 & 0.8396 & 0.8439 & -- & 0.8254 & 0.8310 & 0.7833 & 0.5942 \\
Thursday & 0.7625 & 0.9262 & 0.9377 & 0.8137 & -- & 0.7438 & 0.7148 & 0.5332 \\
Friday & 0.7946 & 0.9378 & 0.9320 & 0.8097 & 0.9327 & -- & 0.8156 & 0.5247 \\
Saturday & 0.8908 & 0.8066 & 0.7821 & 0.7341 & 0.7895 & 0.8490 & -- & 0.6739 \\
Holidays & 0.7142 & 0.6000 & 0.5833 & 0.5290 & 0.6007 & 0.6007 & 0.7659 & -- \\
\hline
\end{tabular}
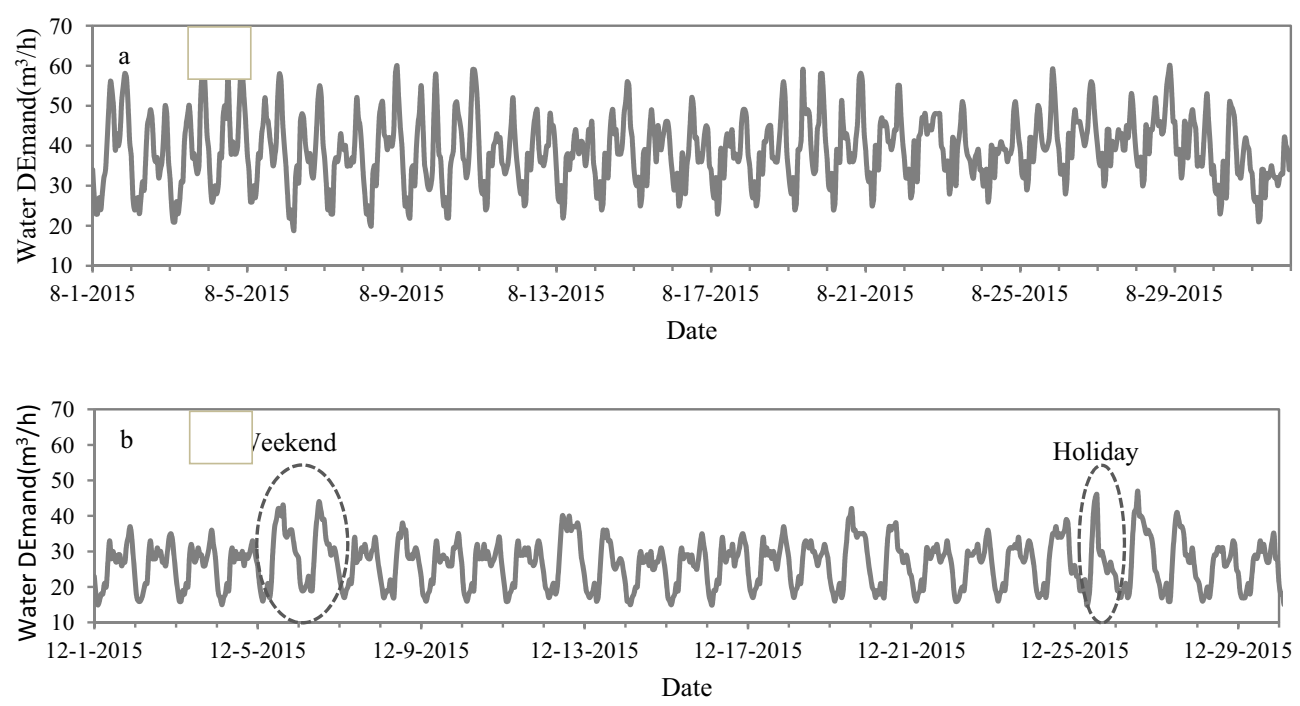

Fig. 9. Daily consumption patterns of WDa for a warm month (a) for August 2015 and daily consumption patterns of WDa for a cold month (b).

were determined using the AIC quality criterion. The best model found was the seasonal ARIMA with the parameters $(1,0,2)(2,0$, 2)24. Fig. 10 shows an example of the typical diagnostic diagrams used for the ARIMA model.

Fig. 11 shows an abrupt decrease of the MAPE until reaching the value of the 336 observations, as similar to the benchmark result. Then, the errors gradually decrease due to the overtraining of the model. However, the execution time increases proportionally with the size of observations not justifying the use of larger observations. The execution time and CPU effort should also be considered in choosing the forecasting model and its input data for the case of real-time forecasts of large size water supply networks, where more than a hundred points must be constantly forecasted. Therefore, $336 \mathrm{~h}$ of observations were used for defining the ARIMA model without day classification. In the model with day classification, a sensitivity analysis was performed similar to the previous one and a sample of $72 \mathrm{~h}$ ( 3 common days) was selected for training the model.

The heuristic forecasting model used allows the generation of short-term forecasts using a set of historical data of $1680 \mathrm{~h}$ to determine the average flow rate used in its calculations and $72 \mathrm{~h}$ of the typical day to calculate the time factors that allow defining the pattern of consumption in the forecast. This number of hours corresponds to 3 typical days according to this classification (working days, weekends and holidays).

All datasets were divided into sub-datasets containing 336 training hours and the respective forecast computation for the next $48 \mathrm{~h}$ for validation.
To determine the parameters of adaptive weights $k$, a sensitivity analysis was performed for different temporalities: from 1 to 56 days before the day of forecast. The parameters $k$ were calculated by minimizing the MAPE error function from the predicted and observed values of the water demand in the historical data. Once the parameters are obtained, these values are used for the calculations of the forecast of the following $48 \mathrm{~h}$. It was found that the optimal time for the calculation of the $k$ parameters is one day. Fig. 12 shows the evolution of the mean MAPE for several amounts of historical data for the cases of WD2 and WDa.

Once the adaptive weights $k$ were obtained, the forecasts of the water demands and the MAPE have been calculated for a period of $48 \mathrm{~h}$. This process was carried out for all time series studied throughout the set of observations and corresponds to a year of study. The results for the validation forecasts of $48 \mathrm{~h}$ (30-31 July 2013) and (29-30 December 2015) are shown in Figs. 13 and 14 for WD2 and WD4 water demands, respectively.

Table 4 provides the forecasting accuracy measurements of all models used for each water demand data set. The values shown are the averages obtained for the entire time interval of each series.

It can be clearly seen in Table 4 that the PAWS model is the best in all the time series studied. The Pearson correlation coefficients show a high correlation between the observed values and the forecasts for all data sets, excepted for the WD16, where there are a high variability and heterogeneity of consumption, making it difficult to capture the patterns by the models. This difficulty in 

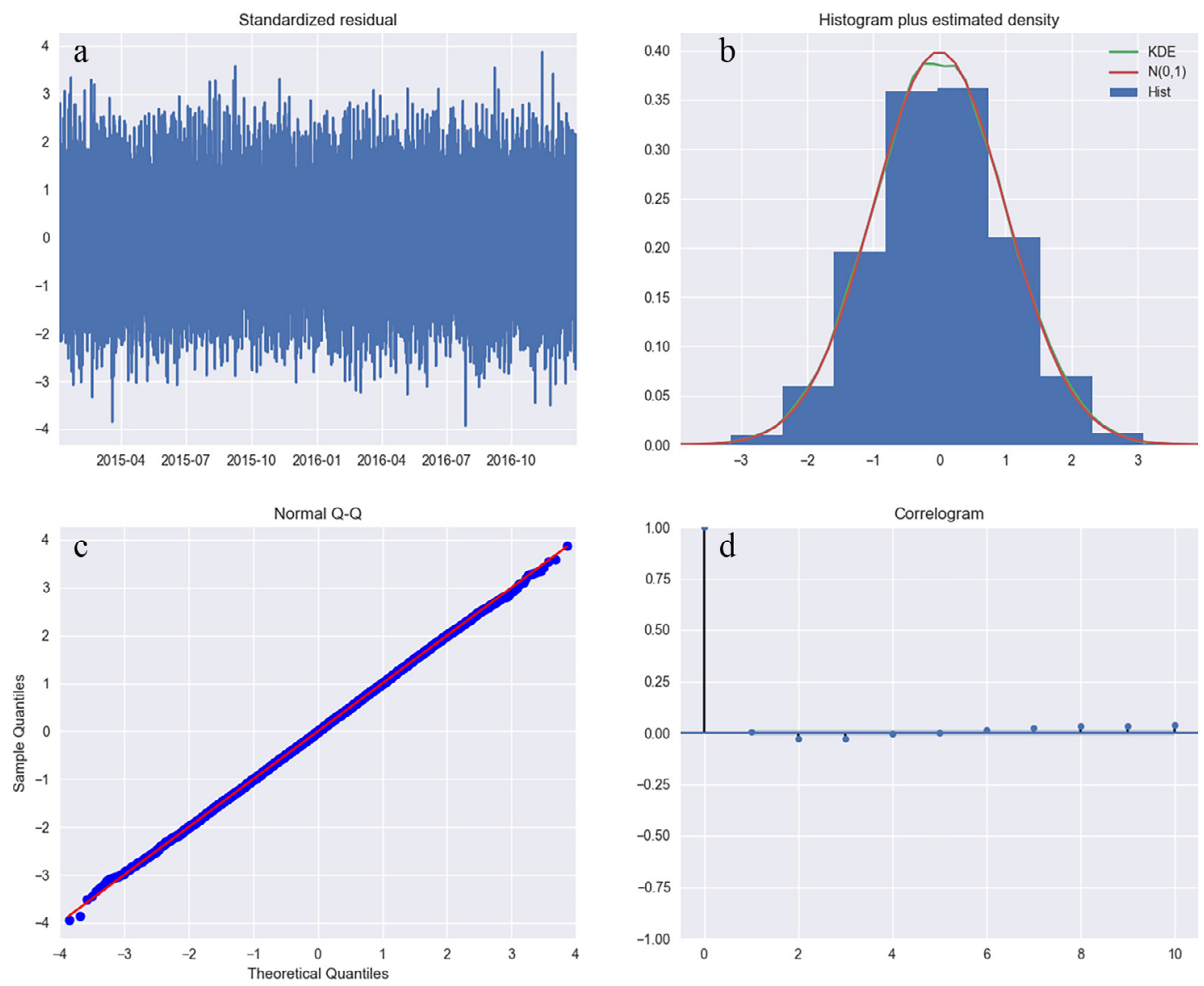

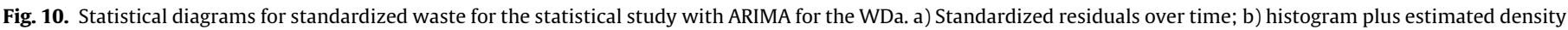
of standardized residuals, along with a $\operatorname{Normal}(0,1)$ density plotted for reference; c) normal Q-Q plot, with Normal reference line; d) correlogram PAF.

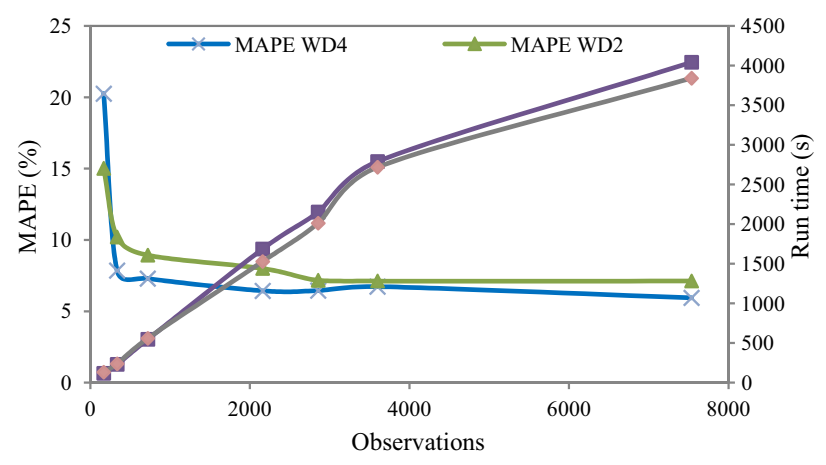

Fig. 11. Evolution of the MAPE error according to the number of observed values and runtime for WD2 and WD4 for model ARIMA without a cluster.

performing good forecasts is seen in the MAPE found for this series presenting values close to $38 \%$ (ARIMA-WD16).

The mean average adaptive parameters obtained for the different series show that the heuristic model presents the forecast closest to the one observed, however, the weighting methodology allows to improve even more the forecast. According to the simulation results, it can be observed that the averages of the $k$ weighting parameters vary from 0.074 to 0.542 and are adapted according to the performance of each forecast model in its most recent history.

The classification of the days performed to calculate the ARIMA clustering model according to the typical day does not always yield

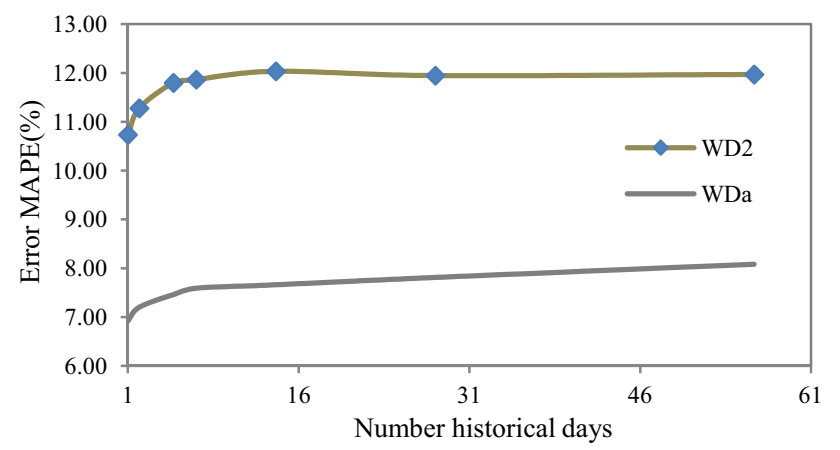

Fig. 12. Sensitivity analysis of MAPE error based on the number of historical data for WD2 and WDa for the calculation of the adaptive weight parameters $k$.

better results than the calculation made with the raw sets, although the values are very similar.

It can be seen that the PAWS model improves the performance in 4 of the 6 time series studied according to the MAPE, achieving reductions of up to $15.96 \%(\mathrm{WDb})$ with respect to the value of the closest forecast (MSE weights).

Figs. 15 and 16 show the forecasts of water demands for the two time series WD4 and WDa for the last $48 \mathrm{~h}$ of each series. When comparing the observed values with each of the models proposed separately, it is evident that each one follows the trend of the cyclic demand pattern, with the PAWS model having the most accurate relationship. 


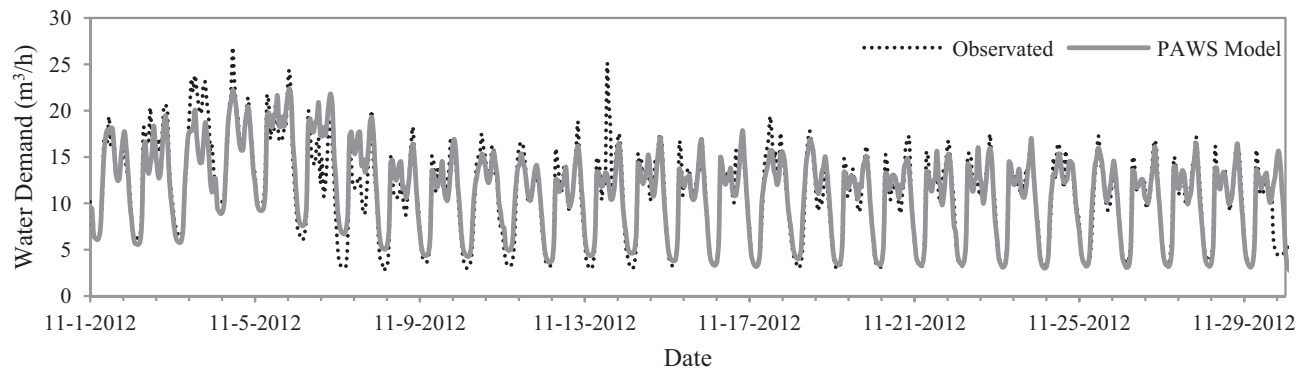

Fig. 13. Water demand forecasts for WD2 for the month of November 2012 calculated at every $48 \mathrm{~h}$.

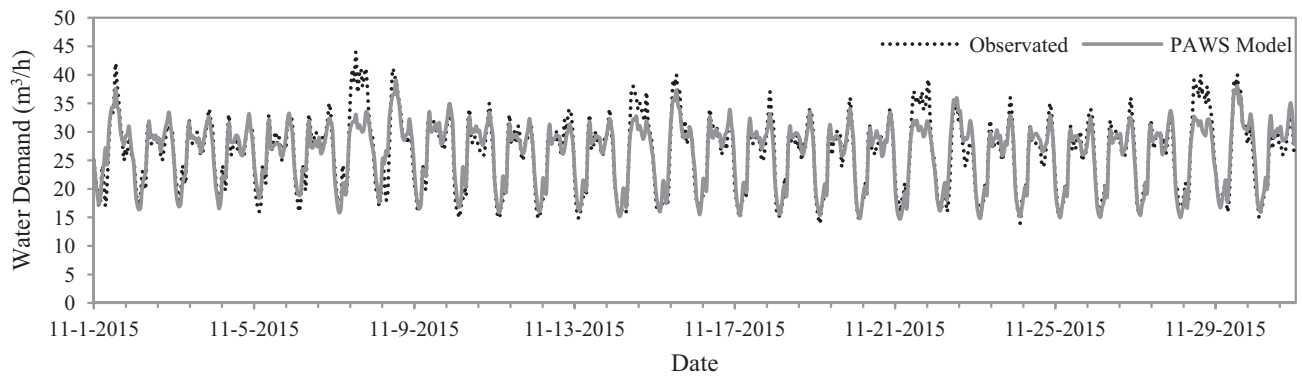

Fig. 14. Water demand forecasts for WDa for the month of November 2015 calculated at every $48 \mathrm{~h}$.

Table 4

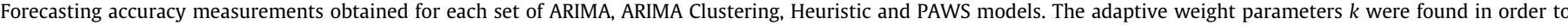
minimize the MAPE in the last $24 \mathrm{~h}$.

\begin{tabular}{|c|c|c|c|c|}
\hline Dataset & Forecasting Model & Adaptive weight parameters $k$ & $\mathrm{R}^{2}$ & MAPE (\%) \\
\hline \multirow[t]{6}{*}{ WD2 } & ARIMA & 0.285 & 0.905 & 14.455 \\
\hline & ARIMA clustering & 0.136 & 0.907 & 16.002 \\
\hline & Heuristic & 0.579 & 0.927 & 12.764 \\
\hline & Mean weights & - & 0.932 & 12.960 \\
\hline & MSE Weights & - & 0.938 & 10.91 \\
\hline & PAWS & - & 0.937 & 10.73 \\
\hline \multirow[t]{6}{*}{ WD4 } & ARIMA & 0.225 & 0.913 & 12.72 \\
\hline & ARIMA clustering & 0.374 & 0.947 & 11.18 \\
\hline & Heuristic & 0.401 & 0.947 & 10.82 \\
\hline & Mean weights & - & 0.961 & 8.730 \\
\hline & MSE Weights & - & 0.968 & 8.562 \\
\hline & PAWS & - & 0.959 & 9.56 \\
\hline \multirow[t]{6}{*}{ WD16 } & ARIMA & 0.235 & 0.864 & 37.603 \\
\hline & ARIMA clustering & 0.273 & 0.869 & 36.542 \\
\hline & Heuristic & 0.492 & 0.885 & 31.898 \\
\hline & Mean weights & - & 0.821 & 35.325 \\
\hline & MSE Weights & - & 0.874 & 34.687 \\
\hline & PAWS & - & 0.899 & 31.049 \\
\hline \multirow[t]{6}{*}{ WDa } & ARIMA & 0.318 & 0.897 & 8.824 \\
\hline & ARIMA clustering & 0.158 & 0.873 & 9.481 \\
\hline & Heuristic & 0.523 & 0.923 & 8.054 \\
\hline & Mean weights & - & 0.838 & 9.578 \\
\hline & MSE Weights & - & 0.839 & 7.334 \\
\hline & PAWS & - & 0.932 & 6.915 \\
\hline \multirow[t]{6}{*}{ WDb } & ARIMA & 0.298 & 0.850 & 9.340 \\
\hline & ARIMA clustering & 0.021 & 0.687 & 9.678 \\
\hline & Heuristic & 0.681 & 0.902 & 7.847 \\
\hline & Mean weights & - & 0.862 & 9.125 \\
\hline & MSE Weights & - & 0.921 & 6.321 \\
\hline & PAWS & - & 0.902 & 7.522 \\
\hline \multirow[t]{6}{*}{ WDc } & ARIMA & 0.502 & 0.752 & 6.931 \\
\hline & ARIMA clustering & 0.074 & 0.710 & 7.037 \\
\hline & Heuristic & 0.425 & 0.756 & 7.044 \\
\hline & Mean weights & - & 0.801 & 7.155 \\
\hline & MSE Weights & - & 0.799 & 7.233 \\
\hline & PAWS & - & 0.782 & 6.614 \\
\hline
\end{tabular}




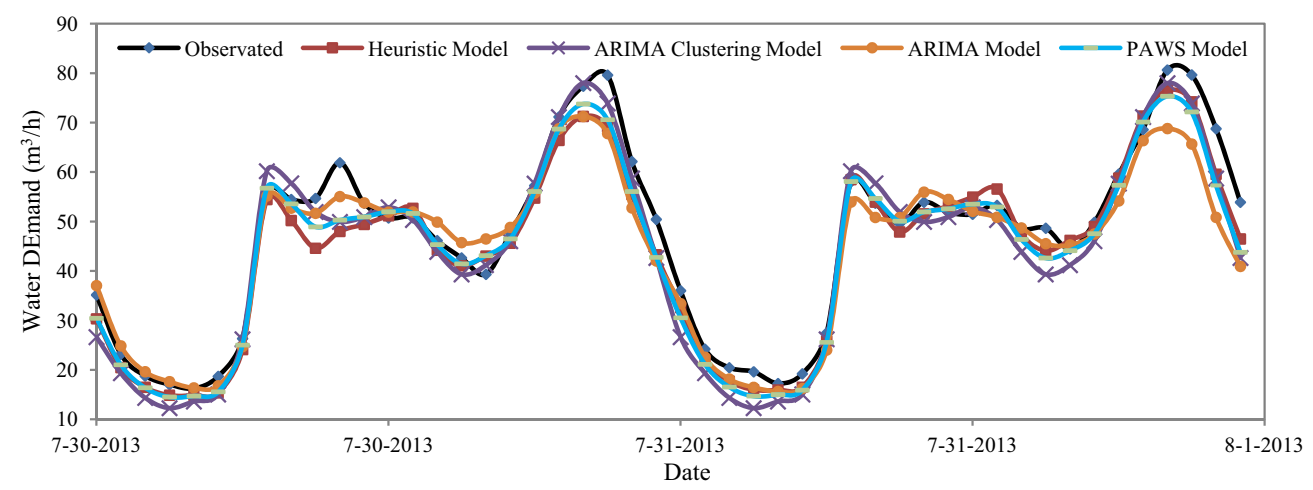

Fig. 15. Forecasts of the water demands obtained by the ARIMA, ARIMA Clustering, Heuristic and PAWS models for the 48-h WD4 dataset.

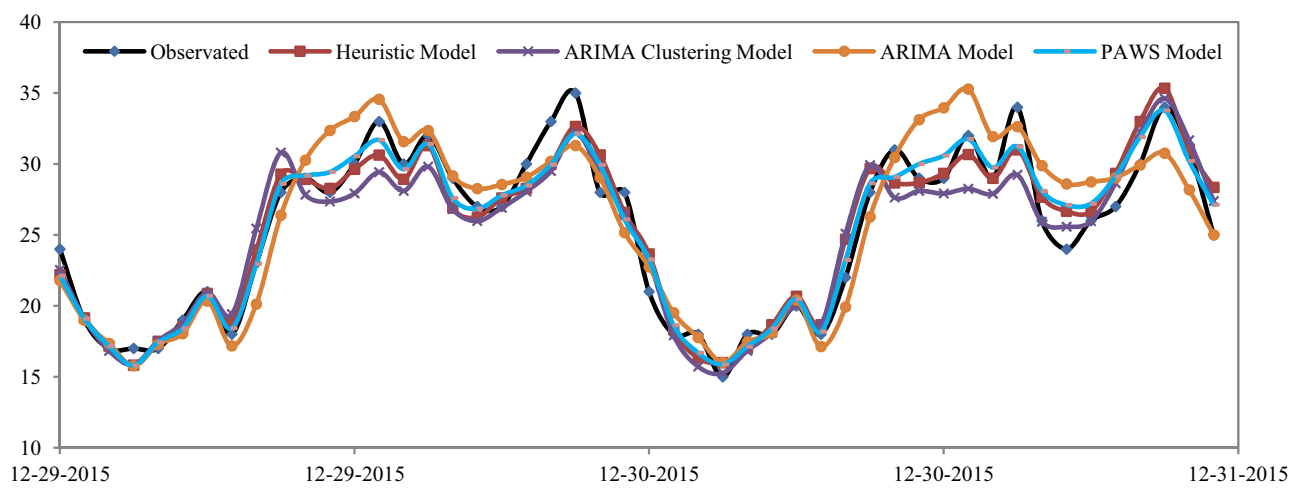

Fig. 16. Forecasts of the water demands obtained by the ARIMA, ARIMA Clustering, Heuristic and PAWS models for the 48-h WDa dataset.

\section{Conclusions}

Using the historical drinking water consumption data and the calendar days as the only data entry, the performance of the proposed PAWS prediction model produced a MAPE that improves the use of individual forecast models. The proposed model yields acceptable prognostic values in relation to the random behavior of the data used.

Individually, the best performing model is the heuristic model, however, the parallel combination with other models and an efficient weighting calculation methodology increase the overall performance of the method.

In general, for the different sets of water demands, it was shown that parallel adaptive weights found by optimization (in autoregressive models and the heuristic model) improved the individual models by predicting the demand for drinking water for one day.

For the time series studied, it was shown that the parallel combination of different forecasting models of water demand improves overall performance, reducing the error $15.96 \%$. This improvement may be relevant when prediction accuracies are needed when short-term forecasting is used for optimal control of drinking water supply systems.

\section{Acknowledgements}

The authors gratefully acknowledge the financial support of the Portuguese Foundation for Science and Technology (FCT) under the projects P2020-PTDC/EMS-TEC/6400/2014 (POCI-01-0145-FEDER016876) by UE/FEDER through the program COMPETE 2020 and UID/EMS/00481/2013-FCT under CENTRO-01-0145-FEDER022083.

\section{Appendix A}

The function used as benchmark simulates the consumption of drinking water with a daily pattern that repeats infinitely. The adopted pattern has a valley during the night, a peak in the morning and another in the evening. Additionally, weekly and monthly seasonalities are added. The added random noise has the purpose of generating different daily patterns. The function used in this process is defined as

$D(t)=50+\frac{\sum_{i=1}^{5} f_{i}(t)}{5}+\operatorname{GAUSSIAN}(0,2)$,

where

$f_{i}(t)=\alpha_{i} \sin \left(\beta_{i}+\frac{2 \pi}{\gamma_{i}} t\right)$.

$\alpha=\{30,30,30,20,10\} ; \beta=\{3,-3,10,-3,0\} ; \gamma=\{12,24,24$, $168,744\}$ and GAUSSIAN (0.2) represents a Gaussian noise with mean 0 and standard deviation 2 . The functions $f_{1}, f_{2}$ and $f_{3}$ simulate the daily behavior for periods of 12,24 and $24 \mathrm{~h}$, respectively, and the functions $f_{4}$ and $f_{5}$ represent the weekly and monthly patterns, defined by their periods of 168 and $744 \mathrm{~h}$.

\section{References}

Adamowski, J., Fung Chan, H., Prasher, S.O., Ozga-Zielinski, B., Sliusarieva, A., 2012. Comparison of multiple linear and nonlinear regression, autoregressive integrated moving average, artificial neural network, and wavelet artificial neural network methods for urban water demand forecasting in Montreal, Canada. Water Resour. Res. 48 (1)

Alvisi, S., Franchini, M., Marinelli, A., 2007. A short-term, pattern-based model for water-demand forecasting. J. Hydroinf. 09 (1), 39-50. 
Babel, M.S., Shinde, V.R., 2011. Identifying prominent explanatory variables for water demand prediction using artificial neural networks: a case study of bangkok. Water Resour. Manage. 25 (6), 1653-1676.

Bai, Y., Chenb, Z., Xiec, J., Li, C., 2015. Daily reservoir inflow forecasting using multiscale deep feature learning with hybrid models. J. Hydrol. 532, 193-206.

Bakker, M., Van Duistc, H., Van Schagenb, K., Vreeburgd, J., Rietvelda, L., 2013. Improving the performance of water demand forecasting models by using weather input. 12th International Conference on Computing and Control for the Water Industry, CCWI2013. Procedia Engineering (70): 93-102.

Bakker, M., Vreeburg, J.H.G., Van Schagen, K.M., Rietveld, L.C., 2013b. A fully adaptive forecasting model for short-term drinking water demand. J. Environ. Modell. Soft. 41, 141-151.

Bates, J., Granger, C., 1969. The Combination of Forecasts. Operat. Res. Q. 20, 451468.

Bennett, N.D., Croke, B.F., Guariso, G., Guillaume, J.H., Hamilton, S.H., Jakeman, A.J., Marsili-Libelli, S., Newham, L.T., Norton, J.P., Perrin, C., Pierce, S.A., Robson, B., Seppelt, R., Voinov, A.A., Fath, B.D., Andreassian, V., 2013. Characterising performance of environmental models. Environ. Modell. Soft. 40, 1-20.

Box, G.E.P., Jenkins, G.M., 1976. Time Series Analysis: Forecasting and Control. Holden-Day, San Francisco.

Caiado, J., 2010. Performance of combined double seasonal univariate time series models for forecasting water demand. J. Hydrol. Eng. 15 (3), 215-222.

Candelieri, A., 2017. Clustering and support vector regression for water demand forecasting and anomaly detection. Water 9, 224.

Coelho, B., 2016. Energy efficiency of water supply systems using optimisation techniques and micro-hydro turbines. Ph.D. Thesis. Universidade de Aveiro. Aveiro, Portugal.

Franklin, S., Maidment, D., 1986. An evaluation of weekly and monthly time series forecasts of municipal water use. Water Resour. Bull. 22, 611-621.

Ghiassi, G., Zimbra, D.K., Saidane, H., 2008. Urban water demand forecasting with a dynamic artificial neural network model. J. Water Resour. Plann. Manage. 134 (2), 138-146.

Herrera, M., Torgo, L., Izquierdo, J., Pérez-García, R., 2010. Predictive models for forecasting hourly urban water demand. J. Hydrol. 387, 141-150.

Homwongs, C., Sastri, T., Foster, J.W., 1994. Adaptive fore-casting of hourly municipal water consumption. J. Water Resour. Plann. Manage. 120 (6), 888905.

Hyndman, R., Athanasopoulos, G., 2013. Forecasting: principles and practice. OTexts, Available from: <http://otexts.org/fpp/>.
Jowitt, P.W., Chengchao, X., 1992. Demand forecasting for water distribution systems. Civil Eng. Syst. 9 (2), 105-121.

Maidment, D.R., Parzen, E., 1984a. Time patterns of water use in six Texas cities. J Water Resour. Plann. Manage. 110 (1), 90-106.

Miaou, Shaw-Pin, 1990. A class of time series urban water demand models with nonlinear climatic effects. Water Resour. Res. 26, 169-178.

Msiza, I., Nelwamondo, F., Marwala, T., 2008. Water demand prediction using artificial neural networks and support vector regression. J. Comput. 3 (11), 1-8.

Quevedo, J., Puig, V., Cembrano, G., Blanch, J., Aguilar, J., Saporta, D., Benito, G., Hedo, M., Molina, A., 2010. Validation and reconstruction of flow meter data in the Barcelona water distribution network. Control Eng. Pract. 18 (6), 640-651.

Sampathirao, A., Grosso, J., Sopasakis, P., Ocampo-Martinez, C., Bemporad, A., Puig V., 2014. Water demand forecasting for the optimal operation of large-scale drinking water networks: The Barcelona Case Study. IFAC Proceedings Volumes 47 (3):10457-10462.

Santos, C., Filho, A., 2014. Water Demand Forecasting Model for the Metropolitan Area of São Paulo, Brazil. Water Resourc. Manage. 28, 4401-4414.

Schütze, M., Campisano, A., Colas, H., Schilling, W., Vanrolleghem, P., 2004. Realtime control of urban wastewater systems-where do we stand today? J. Hydrol. 299 (3-4), 335-348.

Shvartser, L., Uri, S., Mordechai, F., 1993. Forecasting hourly water demands by pattern recognition approach. ASCE J. Water Resour. Plann. Manage. 119, 611627.

Smith, J., 1998. A model of daily municipal water use for short-term forecasting. Water Resour. Res. 24 (2), 201-206.

Stock, J., Watson, M., 1999. A Comparison of Linear and Nonlinear Univariate Models for Forecasting Macroeconomic Time Series. Oxford University Press, Oxford.

Tabesh, M., Dini, M., 2009. Fuzzy and neuro-fuzzy models for short-term water demand forecasting in Tehran. Iran. J. Sci. Technol. Trans. B, Eng. 33 (B1), 61-77.

Vito De, S., Massera, E., Piga, M., Martinotto, L., Francia Di, G., 2008. On field calibration of an electronic nose for benzene estimation in an urban pollution monitoring scenario. Sensor. Actuat. B-Chem. 129 (2), 750-757.

Winkler, R., Makridakis, S., 1983. The Combination of Forecast. J. Roy. Stat. Soc Series A 146 (2), 150-157.

Zhang, G., 2003. Time series forecasting using a hybrid ARIMA and neural network model. Neurocomputing 50, 159-175.

Zhou, S., McMahon, T., Walton, A., Lewis, J., 2002. Forecasting operational demand for an urban water supply zone. J. Hydrol. 259, 189-202. 